\title{
Niveaux d'eau à prendre en compte pour la conception et la réali- sation des ouvrages de génie côtier et portuaire
}

\author{
Daniel Caminade ${ }^{1}$, Daniel Levacher ${ }^{2}$ \\ ${ }^{1}$ Maître de conférences, ISA-BTP, Laboratoire des Sciences Appli- \\ quées au Génie Civil (LASAGEC), Parc de Montaury, 64600 Anglet. \\ ${ }^{2}$ Professeur des Universités, Université de Caen, Morphodynamique \\ Continentale et Côtière (M2C), 24 rue des Tilleuls, 14000 Caen.
}

Résumé

Cet article présente une liste des divers éléments qui ont une influence sur le niveau de la mer ainsi que leur évolution prévisible à court ou moyen terme. Il propose une méthodologie pour associer ces divers facteurs en vue de la détermination des niveaux hauts et bas, normaux ou exceptionnels, à retenir pour les études de conception et de réalisation des ouvrages et des aménagements portuaires ou côtiers en tenant compte des risques. Enfin, il présente deux exemples de caícul de ces niveaux dans une région soumise à des cyclones.

\begin{abstract}
The knowledge of mean sea level is an important feature in maritime structures design. The present paper gives a list of the various factors which have some effects on sea level. A method for determining low and high sea levels for normal or exceptional conditions is shown out. These values must be correlated with the swell height in order to piecise the risk of failure of coastal structures. Eventually, two examples of sea levels calculation are given for an area submitted to hurricanes.
\end{abstract}

\section{Introduction}

Des événements récents, largement relayés par les médias, tels que les inondations du Vietnam central dans la région de Hué au milieu de l'année 1999 ou plus récemment les inondations en France dans les régions proches du Golf du Lion en novembre 1999 ou celles de la Gironde en décembre, pour ne citer qu'elles, ont mis en évidence le rôle essentiel du niveau "anormalement" élevé de la mer.

On peut expliquer l'importance des dégâts par l'action conjuguée de divers paramètres, outre la violence des précipitations: 
- l'imperméabilisation des terres en France, qui accrôt le volume d'eau à récupérer dans un laps de temps plus court car la vitesse de ruissellement est plus rapide,

- la réduction de la section des rivières et des fleuves, notamment par la disparition ou l'encombrement du lit majeur,

- l'élévation du niveau de la mer, qui en diminuant la pente d'eau réduit considérablement le débit des cours d'eau et donc les capacités d'évacuation des eaux terrestres déjà surabondantes, sans compter l'effet du vent qui peut avoir une influence dans le même sens.

La connaissance des niveaux prévisibles tant dans les conditions normales qu'exceptionnelles, revêt donc une importance capitale dès lors que l'on s'intérésse aux divers aspects du génie côtier: ouvrages de protection contre la mer et contre la houle (détermination de la cote d'arase ou étude de la stabilité) pour lesquels le niveau haut est déterminant, mais aussi pour les ouvrages de prise d'eau pour lesquels c'est le niveau bas qui peut s'avérer important. Cet article propose une analyse des divers paramètres à prendre en compte pour la détermination de ces niveaux tout en essayant de préciser ceux qui ont une corrélation forte avec les houles auxquelles les ouvrages sont susceptibles d'être exposés.

\section{Niveau moyen des océans}

Pour pouvoir estimer les niveaux que peut atteindre la mer en un endroit donné, il est nécessaire de se fixer au préalable un niveau de référence que l'on espère pouvoir considérer comme fixe dans le temps. En France, traditionnellement on retient en général, le zéro NGF fixé par l'IGN. Ce niveau est, en fait, un niveau marin mesuré à Marseille, dont on sait qu'il a largement évolué par le passé que ce soit en Atlantique (A) ou en Méditerranée (M) (Fig.1 d'après TEISSON [6]). Les méthodes de mesure par satellite, actuellement développées apportent d'incontestables améliorations. Ensuite, on utilise généralement une référence locale qui est le zéro hydro, considéré comme étant la cote des plus basses mers

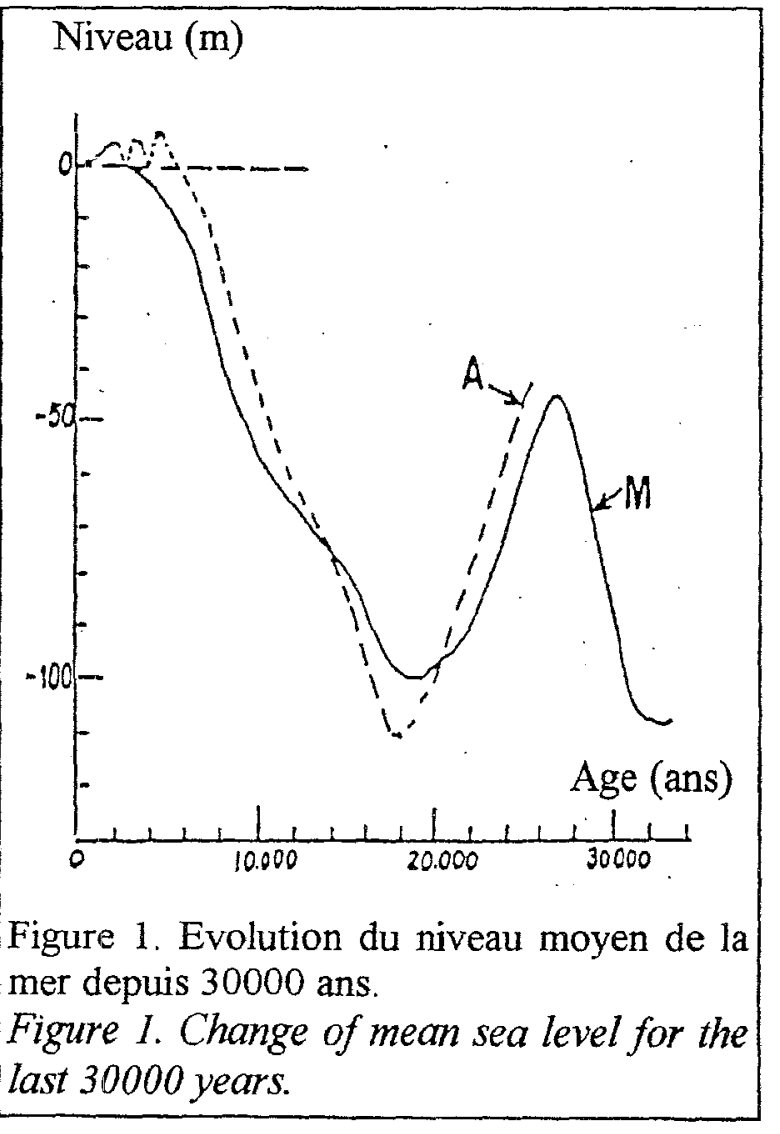


En fait, il vaudrait mieux parler du mouvement relatif de la terre et de la mer en un endroit donné. En effet, si l'on se situe à un endroit de l'écorce terrestre qui s'enfonce sous une plaque, il est évident qu'en cet endroit le niveau apparent de la mer est en train de monter et réciproquement. D'autres facteurs jouent en faveur d'une montée des océans tels que l'érosion terrestre : les éléments détritiques transportés par les fleuves jusqu'à la mer vont combler les fosses océaniques. Mais a contrario de nombreux mouvements et phénomènes terrestres ou sous-marins provoquent des surélévations locales, telles que l'émergence des volcans.

De nombreuses publications font actuellement état d'une montée du niveau des océans, due à de multiples facteurs dont un éventuel réchauffement du globe qui entraînerait une dilatation de l'eau de mer et une fonte des glaces, notamment dans les régions polaires. Les experts sont assez partagés sur l'évolution du niveau des eaux dans un avenir proche, à l'échelle humaine, compte-tenu de la diversité des scénarii concernant les variations dues à la température, mais il semblerait qu'il y ait un consensus pour évaluer la montée des eaux dans nos régions, Océan Atlantique et Mer Méditerranée, à une valeur comprise entre $50 \mathrm{~cm}$ et $1 \mathrm{~m}$ pour le siècle à venir. La figure 2 illustre cette évolution possible à partir de 1980 , dans le cas d'une élévation modérée de la température (d'après Oerlemans 1989 cité par TEISSON [6]).

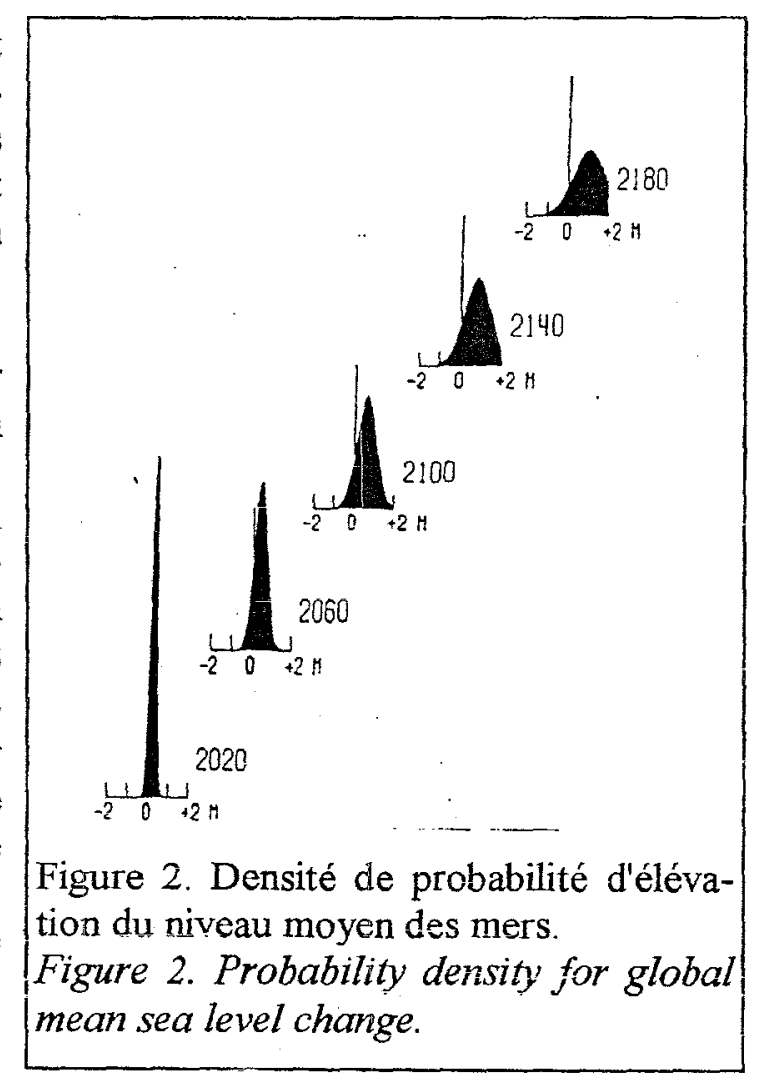

\section{La marée}

La marée est le principal phénomène qui induit de fortes variations du niveau des plans d'eau, mais heureusement ces variations peuvent être prévues et calculées avec une bonne précision. Il n'y a, a priori, pas à craindre un bouleversement de ce phénomène au large des côtes, dans un avenir proche car les phénomènes qui lui donnent naissance sont stables : révolution des astres et dimensions des océans.

Par contre, l'onde de marée en se propageant est susceptible de se déformer surtout lorsqu'elle aborde les zones côtières. Tout d'abord, cette variation peut être due à des travaux locaux d'aménagement: l'approfondissement d'un estuaire pour améliorer les conditions d'accès des navires a pour conséquence de mieux faire pénétrer la marée dans la partie maritime de l'estuaire. Les marées hautes seront 
plus hautes (avec les risques de submersion des ouvrages de protection) et les marées basses plus basses. Lors de l'aménagement de l'estuaire de la Loire pour la création du terminal méthanier de Montoir, des études sur modèles avaient été effectuées et elles avaient notamment conclu qu'il n'y avait pas de risque de voir le coin salé remonter jusqu'aux prises alimentant en eau douce la région de Nantes. Mais l'abaissement du niveau des plus basses eaux a entraîné des incidents sur les prises d'eau de la centrale de Cordemais et l'EDF a été conduite à modifier cellesci.

D'autres facteurs tels que les vents, la houle ou les variations de pression atmosphériquue, peuvent avoir des conséquences sur la vitesse de propagation de l'onde de marée qui peut alors subir des décalages en hauteur et en temps. Le retard ou l'avance de la marée peut facilement être mis en évidence lors de l'analyse de la courbe de marée notamment à basse et haute mers (Fig. 3). Ces décalages dans le temps se traduisent pour l'exploitation des ports par des variations de niveau plus importantes que les décotes ou surcotes. Au Havre il n'est pas rare d'observer à mi-marée un décalage de l'ordre du mètre alors qu'à l'étale celui-ci est inférieur à $30 \mathrm{~cm}$.

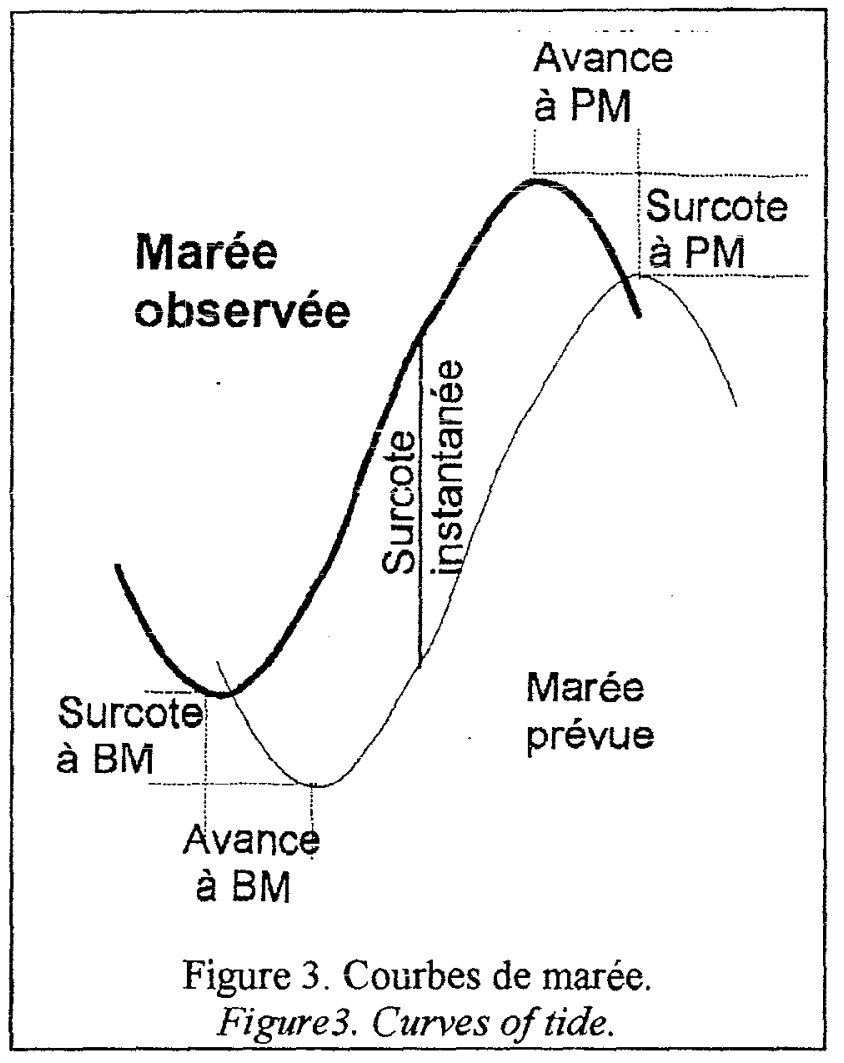

igure 3. Courbes de marée. Figure3. Curves of tide.

La mise en évidence des décalages de niveau uniquement dus à la variation de la propagation de l'onde est beaucoup plus difficile à réaliser car ces variations sont masquées par les phénomènes eux-mêmes qui sont à l'origine des surcotes et des décotes. Bien souvent, on les prend en compte globalement.

\section{Les ondes de tempête}

Les tempêtes sont générées au large des côtes par des vents violents qui sont eux-mêmes engendrés par des différences et des gradients de pression importants. La surface des océans se comportant comme un véritable baromètre, les zones dépressionaires verront une montée du niveau de l'eau qui peut être importante: en septembre 1999, la pression atmosphérique au centre du cyclone Floyd était de $920 \mathrm{hPa}$, ce qui correspond à une surélévation de l'ordre du mètre. A l'inverse les zones de haute pression verront leur niveau s'abaisser créant ainsi une dénivellation donnant naissance à une onde dite de tempête qui va se propager. 
Ces ondes sont à l'origine du "raz de marée" qui a submergé le Sud-Ouest de la Hollande le 30 janvier 1953. Plus près de nous, en novembre 1973, on a enregistré une surcote de $3 \mathrm{~m}$. La propagation de ces ondes a pu être modélisée numériquement, en tenant compte des diverses diffractions, réfractions ou réflexions, ce qui a permis d'expliquer l'importance des surélévations constatées.

Pour se prémunir des effets de tels phénomènes, il est nécessaire de surélever les digues et parfois de construire des barrages anti-tempêtes comme celui érigé sur la Tamise ou celui qui protège les terres inondables de Hollande. Les vannes de ce dernier sont actionnées dès que l'amplitude observée de la marée dépasse de $3 \mathrm{~m}$ les prévisions, soit en moyenne 2 fois par an.

Ces phénomènes sont donc loin d'être exceptionnels et doivent être pris en compte pour l'estimation des niveaux d'eau en vue de la détermination de la cote d'arase des ouvrages.

\section{Paramètres locaux}

Localement, le niveau de l'eau peut être influencé par de nombreux paramètres dont les plus courants sont:

- les variations de la pression atmosphérique,

- le vent local,

- la mer du vent générée localement,

- le débit d'un fleuve, notamment en estuaire,

- les fortes précipitations,

- les oscillations locales du plan d'eau telles que les seiches...

La mesure des différents paramètres associée à une mesure en continu de la hauteur d'eau va permettre après analyse, d'estimer limpact de chaque facteur sur les variations de hauteur du plan d'eau. Cette analyse doit, au préalable à tout calcul, permettre d'identifier les variables indépendantes de celles qui présentent une corrélation forte. C'est seulement après cela que l'on peut procéder à une analyse statistique qui va permettre de prévoir les valeurs à adopter en fonction des périodes de retour retenues pour l'étude du projet.

\section{Corrélation des divers paramètres}

\subsection{Paramètres non liés}

D'une manière générale, il s'agit des paramètres qui sont générés par des phénomènes qui sont indépendants tels que: les variations de niveau d'origine géologique, la marée d'origine astronomique, la houle du large. Ainsi, il n'y a aucune raison de voir apparaître de fortes tempêtes lors de grandes marées. Par contre, localement près des cotes, il peut y avoir une fausse corrélation: le niveau de la mer 
étant plus haut que la moyenne, la houle du large sera moins soumise au déferlement et frappera donc les ouvrages de protection côtière avec une force accrue.

De même, il n'y a pas de corrélation à attendre entre des phénomènes locaux et des modifications lointaines: surcotes et houle du large n'ont, a priori, aucune raison d'être liées. On devra toutefois, lors des études, porter une attention particulière à la possibilité de combinaison de ces phénomènes, eu égard aux conditions de propagation des perturbations générées au large: une forte dépression va créer des ondes de tempête et de la houle qui vont se propager avec leurs vitesses propres. La dépression peut elle-même se propager et en s'approchant des côtes entraîner une surélévation locale du plan d'eau, des vents soufflant du large et de la houle locale (mer du vent). Ce type de phénomène peut aussi se produire lorsque plusieurs dépressions se suivent à intervalle rapproché comme cela s'est passé en France, fin 1999.

La conjugaison de tous ces paramètres a des conséquences importantes sur la tenue des ouvrages côtiers: niveau d'eau élevé et forte houle risquent de mettre en péril les structures de protection d'autant qu'un autre phénomène va encore aggraver les choses. Lors des études entreprises pour la réfection de la digue de Sines (Portugal), le laboratoire de Delft a mis en évidence que pour une même houle significative (Hs) et une même période (Tpic), les spectres les plus agressifs étaient ceux qui représentaient une houle du large associée à une mer du vent locale (spectre sea and sweli).

\subsection{Paramètres liés}

L'exemple type est donné par les cyclones pour lesquels on constate la concomitance de nombreux facteurs qui engendre une forte surélévation du plan d'eau et des houles fortes: dépression importante engendrant des surcotes, des ondes de tempête, des vents, de la houle, précipitations à l'origine des crues...

Dans nos régions, ce sont en général les paramètres locaux qui présentent des corrélations fortes. A titre d'exemple, la Baie de Seine et plus spécialement la région du Havre, présentent deux types de temps assez caractéristiques.

Lorsqu'une dépression arrive par l'ouest, on assiste à la naissance d'une surcote, à l'apparition de vents forts de secteur ouest (sud-ouest à nord-ouest) créant des houles locales qui se propagent d'ouest en est, vents et houles qui poussent la mer vers l'estuaire. Comme ces perturbations sont fréquentes en hiver, elles sont en général accompagnées de pluies violentes qui viennent accroître le débit de la Seine déjà en crue. Toutes les conditions sont donc réunies pour voir le niveau de l'eau monter dans l'estuaire et provoquer des inondations des terrains bas.

A linverse, par régime de vent d'est (en général période de beau temps), on observe des décotes dues à la zone anticyclonique et des vents et houles qui ont plus tendance à "vider" l'estuaire. 
Lors de l'étude du Port du Havre-Antifer (Fig. 4), s'est posée la question des cotes de dragage à adopter. L'enjeu était de taille: $10 \mathrm{~cm}$ de variation du niveau de dragage représentait $500000 \mathrm{~m}^{3}$ de matériaux à enlever sous $30 \mathrm{~m}$ d'eau.

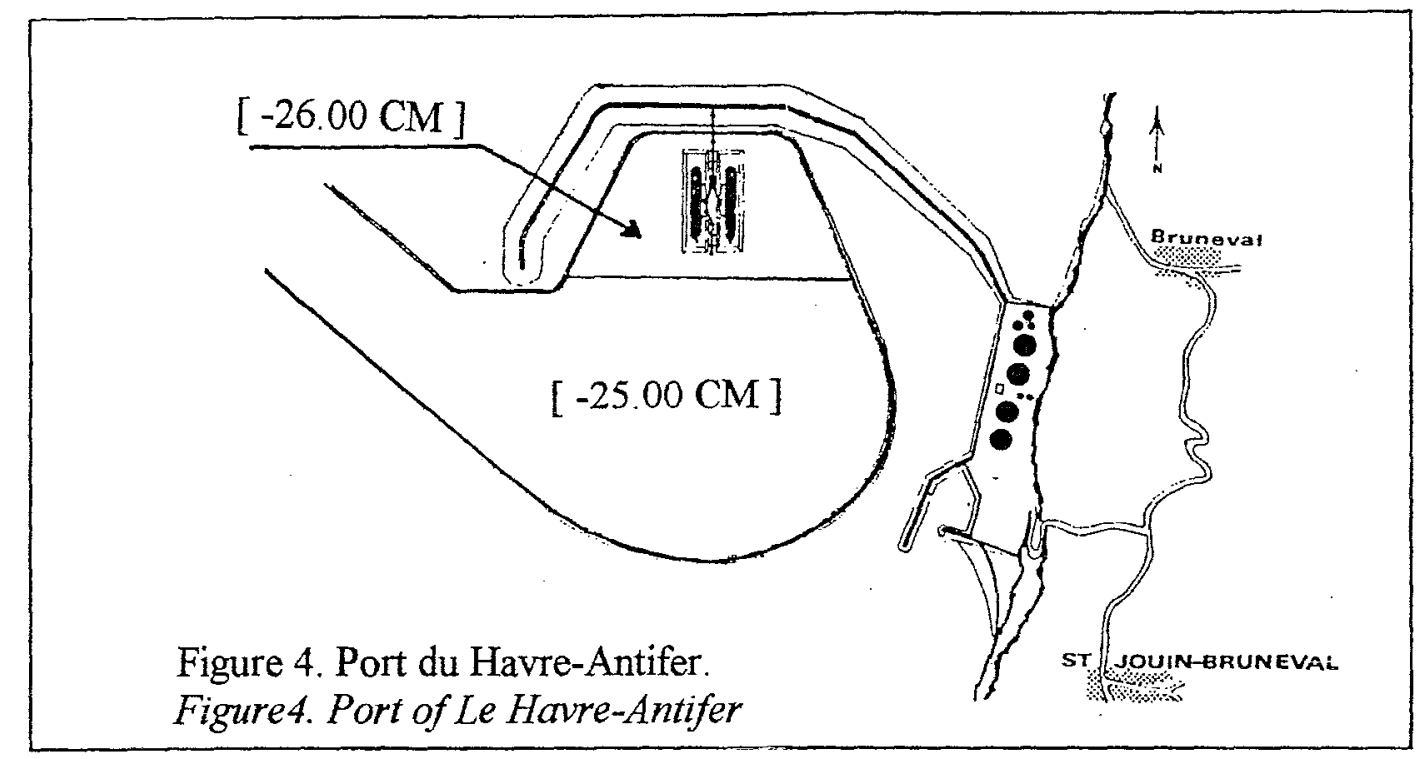

Une étude poussée des conditions d'accès a donc été effectuée. Pãr temps d'ouest, les navires sont soumis à une houle venant de l'arrière qui provoque des mouvements de ceux-ci. Heureusement, l'étude statistique a montré que ces mouvements étaient pour partie compensés par la surélévation du plan d'eau et le pied de pilote pouvait être ainsi réduit. Par temps d'est et dans le chenal d'accès, le pied de pilote devait être plus important car il fallait tenir compte des mouvements du navire sous la houle et des décotes. A l'intérieur du port, le navire étant protégé de la houle on pouvait réduire le pied de pilote. Au total cette étude a permis de définir les cotes de dragage: $(-25.00)$ dans le chenal et le sud de la zone de manoeuvre, $(-26.00)$ dans le nord de la zone, évitant ainsi un dragage général à (-26.00) économisant $2,5 \mathrm{Mm}^{3}$. La zone des postes d'accostage ảraguée à $(-29.80)$ a fait l'objet d'une étude analogue mais plus approfondie.

\section{Méthodologie}

La méthodologie proposée découle de tout ce qui a été exposé ci-avant.

Dans un premier temps, on étudiera les paramètres généraux susceptibles de modifier le niveau des plans d'eau:

- étude géologique pour apprécier s'il y a ou subsidence,

- choix d'un scénario relatif à la montée des eaux et choix des valeurs de surélévation à moyen et long termes,

- étude de la marée et de sa propagation en fonction des aménagements projetés, 
- étude de la possibilité d'apparition des ondes de tempête et de leur propagation en vue de chiffrer les valeurs des surcotes avec leur probabilité d'apparition.

Ensuite, on pourra passer à l'examen des conditions locales et comme nous l'avons dit procéder à une sélection des divers paramètres avant de recourir à une étude statistique dont le but est de fixer les diverses valeurs des surélévations avec leur probabilité d'apparition ou leur période de retour.

Enfin, il faudra combiner toutes ces occurrences pour déterminer les valeurs globales de la surélévation (ou éventuellement la décote) à prendre pour le projet à court, moyen ou long terme. Ces valeurs doivent être assorties d'une estimation de leur fréquence d'apparition en corrélation avec la houle à prendre en compte pour le calcul des ouvrages.

\section{Exempies de calcul}

La Nouvelle Calédonie est composée de la "Grande Terre" et de nombreùses îles dont les Iles Loyauté situées à l'est (Fig. 5).

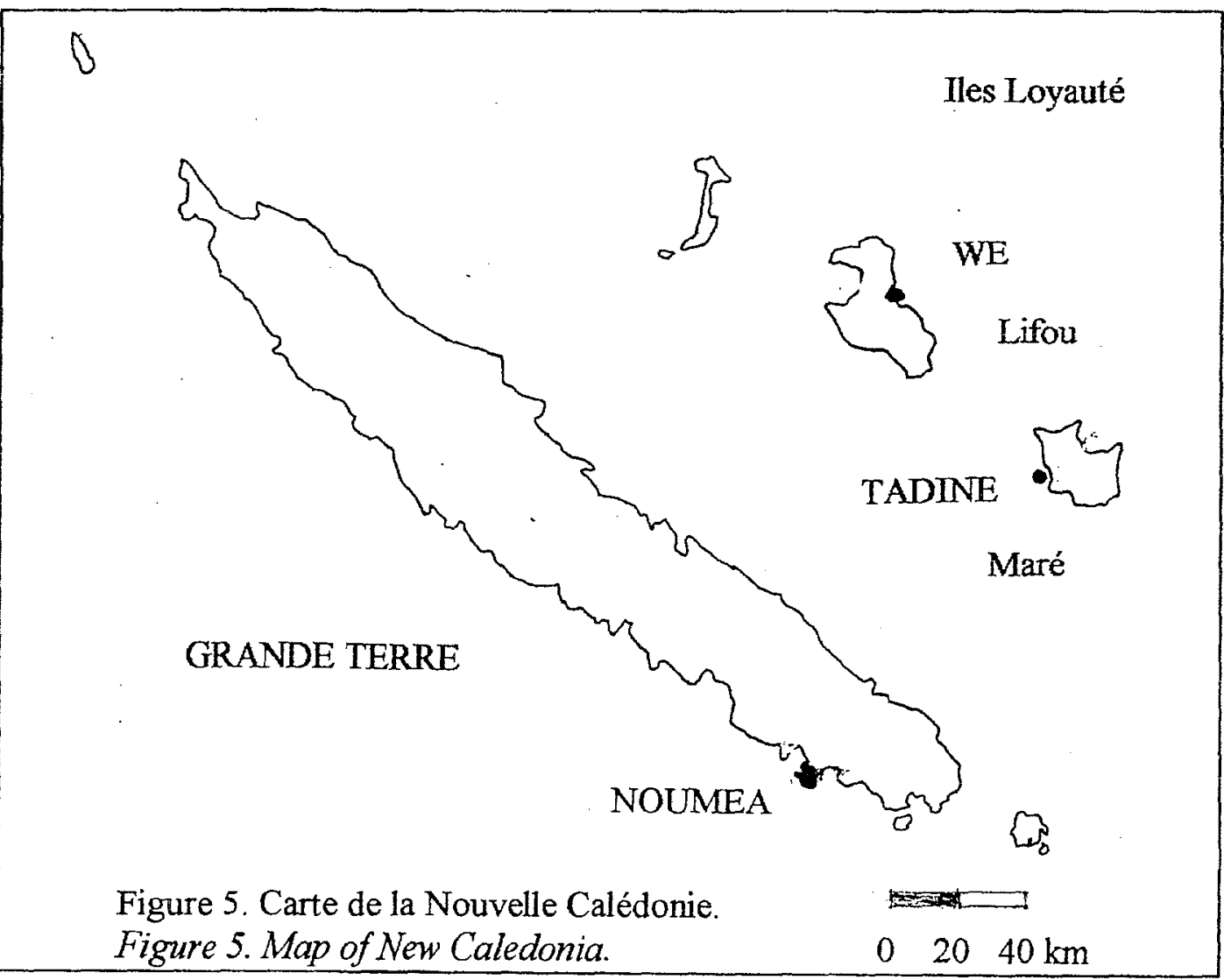

Pour mieux désenclaver ces îles, il a été décidé au début des années 1990 d'en améliorer la desserte maritime notamment en créant des ports abrités des 
houles locales engendrées par les alizés et capables de résister aux houles exceptionnelles lors des cyclones. Pour établir le projet, on a réalisé (CAMNNADE [1] et [2]) une étude des houles pour chacun des deux sites de Wé et de Tadine. Celui de Wé à Lifou, tourné vers le nord-est, est exposé aux houles modérées d'alizé mais est susceptible de subir de plein fouet les tempêtes cycloniques engendrées par un fetch sur le Pacifique sans aucun autre obstacle que les îles du Vanuatu (ex Nouvelles Hébrides) à $300 \mathrm{~km}$ de là. Celui de Tadine à Maré est plus protégé car tourné vers la Grande Terre : 350 jours par an la houle est inférieure à $1,2 \mathrm{~m}$. Mais il peut être soumis à l'action des "coups d'ouest" qui surviennent généralement en août et à celle des cyclones qui arriveraient à se glisser entre la grande Terre et les Iles Loyauté. La fréquence de ces cyclones et la houle générée sont beaucoup plus faibles que pour le port de Wé.

Les deux baies ne sont pas soumises à de forts balancements (résonance des ondes longues et des seiches) du fait de leur configuration.

Lors des cyclones, on constate une forte élévation du niveau des plans d'eau et il a fallu réaliser une étude complète pour chaque site en tenant compte des probabilités d'apparition des cyclones dans le secteur dangereux pour le site concerné. Grâce aux données enregistrées depuis plus de 50 ans, on a pu retracer litinéraire de chaque dépression tropicale ou cyclone qui pouvait poser problème, en suivant son évolution: vitesse et sens de déplacement, champ de pressions, vitesse et direction des vents. Cete étude a permis d'évaluer les variations possibles du niveau du plan d'eau ainsi que les caratéristiques de la houle concomitante. Ces résultats sont rassemblés dans les tableaux ci-dessous.

\begin{tabular}{|c|c|c|c|c|c|}
\hline \multicolumn{7}{|c|}{ PORT DE TADINE } \\
\hline \multicolumn{2}{|c|}{ Période de retour (en années) } & 1 & 10 & 100 & $>1000$ \\
\hline $\begin{array}{c}\text { Caractéristiques } \\
\text { de la houle }\end{array}$ & Creux Hs (en m) & 2.5 & 3.6 & 4.5 & 5.3 \\
\cline { 2 - 6 } & Période Tpic (en s) & 6.5 & 8 & 9 & 9 \\
\hline $\begin{array}{c}\text { Niveaux du } \\
\text { plan d'eau (en mètres) }\end{array}$ & Haut & +1.70 & +2.50 & +2.80 & +3.50 \\
\cline { 2 - 7 } & Bas & +0.20 & +0.20 & -0.20 & -0.20 \\
\hline
\end{tabular}

\begin{tabular}{|c|c|c|c|c|c|}
\hline \multicolumn{7}{|c|}{ PORT DE WE } \\
\hline \multicolumn{2}{|c|}{ Période de retour (en années) } & 1 & 10 & 100 & $>1000$ \\
\hline $\begin{array}{c}\text { Caractéristiques } \\
\text { de la houle }\end{array}$ & Creux Hs (en m) & 3.7 & 5.0 & 6.6 & 7.8 \\
\cline { 2 - 6 } & Période Tpic (en s) & 9 & 11.5 & 14 & 14 \\
\hline $\begin{array}{c}\text { Niveaux du } \\
\text { plan d'eau (en mètres) }\end{array}$ & Haut & +1.70 & +3.00 & +3.50 & +3.50 \\
\cline { 2 - 7 } & Bas & +0.20 & 0.00 & -0.35 & -0.35 \\
\hline
\end{tabular}

Tableau 1. Houles et niveaux d'eau pour l'étude des ports de Wé et de Tadine. Table I. Swell and sea level for We and Tadine ports design. 
Les niveaux extrêmes calculés pour les conditions centennales sont de +2.80 pour Tadine et de +3.50 pour Wé alors que le niveau des marées ne dépasse pas +2.00 . L'étude a limité les surélévations en tenant compte des probabilités d'apparition simultanée des divers phénomènes alors que le cumul brut donnait un niveau maximal possible du plan d'eau de +4.60 pour Tadine et de +4.90 pour Wé.

\section{Conclusion}

Pendant de nombreuses années, les études de stabilité des ouvrages ont été faites en supposant comme fixe le niveau haut des mers, en général pris égal à celui des plus hautes marées. De ce fait, la montée des eaux va avoir pour conséquence une agressivité plus grande de la houle (écrêtement moins efficace sur les petits fonds) et des digues réputées infranchissables vont être soumises à des franchissements qui attaqueront le couronnement et le talus arrière: il y a donc lieu de prévoir dès maintenañt des travaux de réfection, de confortement et de surélévation des digues existantes.

Pour l'avenir, la fixation des conditions dans lesquelles les ouvrages doivent être projetés puis essayés en modèle réduit doivent tenir compte, comme par le passé, des conditions de houle mais aussi des conditions de niveau de la mer.

Le choix de la valeur des paramètres doit, bien entendu, être fait en estimant au mieux les facteurs de risques et les conséquences d'une submersion ou d'une rupture des ouvrages: l'inondation d'une prairie, l'hiver, dans une zone estuairienne ne présente bien entendu pas le même niveau de risque qu'une centrale nucléaire comme l'a montré l'incident de la centrale du Blayais fin 1999.

\section{$\underline{X . \text { Références }}$}

[1] CAMINADE D. (1990) - Desserte maritime des Iles Loyauté. Ile de Lifou Port de Wé. Avant projet détaillé. Rapport d'études. P.A.N. 26p.

[2] CAMINADE D. (1991) - Desserte maritime des Iles Loyauté. Ile de Maré Port de Tadine. Avant projet détaillé. Rapport d'études. P.A.N. 20p

[3] CERC (1984) - Shore Protection Manual. Chap3 pp. 88-128.

[4] LACOMBE H. (1965) - Cours d'océanographie physique. Gauthier-Villars. $392 p$.

[5] SERILLON C. (1999-2000) - Géopolis. Quand la mer monte. Emissions des 26 Décembre 1999 et 2 Janvier 2000.

[6] TEISSON CH. (1992) - Surélévation future du niveau de la mer. Conséquences et stratégies dans l'aménagement du littoral. Rapport d'études et de recherches pour le STCPMVN. 42p. 